\title{
ON TRANSITIVE TRANSLATION FUNCTIONS ${ }^{1}$
}

JAMES W. SCHLESINGER

From the definition ${ }^{2}$ of a fiber space $(E, B, p)$ in terms of a lifting function,

$$
\lambda:\left\{(e, \omega) \in E \times B^{I} \mid p(e)=\omega(0)\right\} \rightarrow E^{I} \text { such that } p \circ \lambda(e, \omega)=\omega,
$$

we are led to a translation function

$$
\tau:\{(e, \omega) \mid p(e)=\omega(0)\} \rightarrow E \quad \text { where } \quad \tau(e, \omega)=\lambda(e, \omega)(1) .
$$

We may also consider the maps $\tau(\omega): p^{-1}(\omega(0)) \rightarrow p^{-1}(\omega(1))$ defined by $\tau(\omega)(e)=\tau(e, \omega)$. A translation function is transitive if $\tau\left(\omega_{1} \cdot \omega_{2}\right)$ $=\tau\left(\omega_{2}\right) \circ \tau\left(\omega_{1}\right)$ where

$$
\omega_{1}(1)=\omega_{2}(0) \text { and }\left(\omega_{1} \cdot \omega_{2}\right)(t)=\left\{\begin{array}{cl}
\omega_{1}(2 t) & \text { for } 0 \leqq t \leqq 1 / 2 \\
\omega_{2}(2 t-1) & \text { for } 1 / 2 \leqq t \leqq 1
\end{array}\right.
$$

The question of when transitive translation functions exist for fiber bundles was raised by W. Hurewicz. The answer this paper supplies is the following.

If a bundle over a finite polyhedron has a structural group $G$ with no small subgroups then it has a transitive translation function if and only if it is equivalent in $G$ to an $H$ bundle where $H$ is a totally disconnected subgroup of $G$.

The central result of this paper is that if $\tau$ is a transitive translation function and the structural group has no small subgroups, then $\tau(\omega)$ depends only on the homotopy class of $\omega$.

All spaces we consider will be Hausdorff spaces; path spaces will have the compact-open topology.

REMARKs. For path spaces one may take as a basis all sets of the form $N=\bigcap_{i=1}^{2^{n}}\left(\left[(i-1) / 2^{n}, i / 2^{n}\right], U_{i}\right)$.

A sequence $\omega_{n}$ converges to the constant $x_{0}$ in the path space $X^{I}$ if and only if every neighborhood $U$ of $x_{0}$ in $X$ contains all but a finite number of the sets $\omega_{n}(I)$. That this is not true for Moore paths pre-

Received by the editors October 17, 1957.

1 This research was supported in part by the United States Air Force under Contract No. AF 18(603)-91, monitored by the Air Force Office of Scientific Research, Air Research and Development Command.

2 W. Hurewicz, On the concept of fiber space, Proc. Nat. Acad. Sci. U.S.A. vol. 41, no. 11, pp. 956-961. 
vents the extension of our results to translation functions defined on Moore paths.

Definitions. A triple $(E, B, p)$ is a regular fiber space if there exists a function $\tau:\{(e, \omega) \mid p(e)=\omega(0)\} \rightarrow E$ such that $p(\tau(e, \omega))$ $=\omega(1)$ and $\tau(e, \omega)=e$ if $\omega(t) \equiv e$. A fiber space is homeomorphic if each of the maps $\tau(\omega)$ is a homeomorphism.

A fiber space is transitive if $\tau\left(\omega_{1} \cdot \omega_{2}\right)=\tau\left(\omega_{2}\right) \circ \tau\left(\omega_{1}\right)$.

In the case of a fiber bundle $(E, B, p, Y, G)$ over a paracompact base space $B$, the Hurewicz uniformization theorem gives the result: $(E, B, p)$ is a regular homeomorphic fiber space and $\tau(\omega)$ may be identified with a member of $G$ through any two applicable coordinate maps. We shall therefore define a translation function $\tau$ for the bundle $(E, B, p, Y, G)$ as a regular homeomorphic translation function for $(E, B, p)$ with the property that $\phi_{i}^{-1}(\omega(1)) \tau(\omega) \phi_{j}(\omega(0)) \in G$ for some pair (and therefore all pairs) of coordinate functions $\phi_{i}, \phi_{j}$ such that $(\omega(1), \omega(0)) \in V_{i} \times V_{j}$.

Definition. $\omega^{*}$ is a reparametrization of $\omega$ if there exists a sense preserving homeomorphism $f$ of the unit interval onto itself such that $\omega^{*}=\omega \circ f$.

LemMA. If $\omega^{*}$ is a reparametrization of $\omega$ and $\tau$ a transitive translation function, then $\tau(\omega)=\tau\left(\omega^{*}\right)$.

From the associativity of the composition of maps we know that $\tau\left(\omega_{1} \cdots \omega_{n}\right)=\tau\left(\omega_{n}\right) \circ \cdots \circ \tau\left(\omega_{1}\right)$ is independent of the bracketting of $\omega_{1} \cdots \omega_{n}$. In this proof we shall approximate $\omega^{*}$ by a path $\omega^{\prime}$ which is obtained from $\omega$ by letting $\omega=\omega_{1} \cdots \omega_{2}^{m}$ in the canonical bracketting (defined below) and $\omega^{\prime}=\omega_{1} \cdots \omega_{2}{ }^{m}$ in another bracketting. The continuity of $\tau$ then gives the result. We shall use the phrase " $\omega=\omega_{1} \cdots \omega_{2}{ }^{n}$ where the bracketting is canonical" to mean $\omega=\bar{\omega} \cdot \tilde{\omega}$ where $\bar{\omega}=\omega_{1} \cdots \omega_{2^{n-1}}$ and $\tilde{\omega}=\omega_{2^{n-1}+1} \cdots \omega_{2}^{n}$ where in each case the bracketting is canonical. The canonical bracketting is defined only when the number of factors is a power of 2 .

Proof of Lemma. Let $\omega^{*}(t)=\omega(f(t))$ and $N$ be a neighborhood of $\omega^{*}$. We may assume $N=\bigcap_{i=1}^{2^{n}}\left(\left[(i-1) / 2^{n}, i / 2^{n}\right], U_{i}\right)$. Let $V_{i}$ be a connected neighborhood of $\left[(i-1) / 2^{n}, i / 2^{n}\right]=C_{i}$ such that $\omega^{*}\left(\mathcal{V}_{i}\right)$ $\subseteq U_{i}$. Choose dyadic rationals $0<m_{1} / 2^{m}<\cdots<m_{2}{ }^{n} / 2^{m}=1$ such that $m_{i} / 2^{m} \in f\left(\mathcal{V}_{i}\right) \cap f\left(\mathcal{V}_{i+1}\right)$. We shall now construct a path $\omega^{\prime}$, which will send $C_{i}$ into $\omega\left(\left[m_{i-1} / 2^{m}, m_{i} / 2^{m}\right]\right) \subseteq \omega^{*}\left(\mathcal{V}_{i}\right) \subseteq U_{i}$.

Let $\omega=\omega_{1} \cdots \omega_{2}^{m}$ where the bracketting is canonical. Let $\omega_{i}^{\prime}$ $=\omega_{m_{i-1}+1} \cdots \omega_{m_{i}}$ where the bracketting is arbitrary. Define $\omega^{\prime}$ $=\omega_{1}^{\prime} \cdots \omega_{2}^{\prime n}$ where the bracketting is canonical. Now $\omega^{\prime}\left(C_{i}\right)=\omega_{i}^{\prime}(I)$ 
$=\omega_{m_{i-1}+1} \cdots \omega_{m_{i}}(I)=\omega\left(\left[m_{i-1} / 2^{m}, \quad m_{i} / 2^{m}\right]\right) \subseteq U_{i} ;$ therefore $\omega^{\prime} \in N$ which proves the result. q.e.d.

In the remainder of this paper we shall assume that the structural group $G$ has the following property:

If $\omega \in G^{I}, \omega(0)=e$ the identity, then either

(1) There exists a sequence $t_{i}$ of reals from the unit interval converging to zero and positive integers $m_{i}$ such that $\left(\omega\left(t_{i}\right)\right)^{m_{i}}$ does not converge to the identity, or

(2) There exists a $t_{0}>0$ such that $\omega(t)=e$ for $t \leqq t_{0}$.

In particular groups with no small subgroups have this property.

THEOREM. If $\tau$ is a transitive lifting function for the bundle $(E, B$, $p, Y, G)$, then the map $\tau(\omega)$ depends only on the homotopy class of $\omega$.

We proceed by a series of lemmas.

LeMma 1. If $\omega_{s}(t)$ is a homotopy of the loop $\omega_{1}(t)$ at $b_{0}$ to the constant loop $\omega_{0}(t)=b_{0}$, then there exists a number $s_{0}>0$ such that $\tau\left(\omega_{8}\right)=e$, the identity, for $s \leqq s_{0}$.

Proof. $\tau\left(\omega_{s}\right)$ is a path in $G$ such that $\tau\left(\omega_{0}\right)=e$. Since condition (2) is our lemma, it suffices to show that condition (1) is impossible. Let $\left(t_{i}, m_{i}\right)$ be a sequence of reals and positive integers, as in condition (1). By the transitivity of $\tau$ we have $\left(\tau\left(\omega_{t_{i}}\right)\right)^{m_{i}}=\tau\left(\omega_{t_{i}}^{m_{i}}\right)$ where $\omega_{t_{i}}^{m_{i}}$ is defined by $\omega^{n+1}=\omega^{n} \cdot \omega$. Since $t_{i}$ converges to zero, we have that $\omega_{t_{i}}^{m_{i}}$ converges to the constant path $\omega_{0}$. Since $\tau$ is continuous, $\tau\left(\omega_{t_{i}}^{m_{i}}\right)$ converges to the identity; thus (1) cannot hold. q.e.d.

If $\omega(t)$ is a path, let $\omega^{-1}(t)$ denote the path defined by $\omega^{-1}(t)$ $=\omega(1-t)$.

Lemma 2. $(\tau(\omega))^{-1}=\left(\tau\left(\omega^{-1}\right)\right)$.

Proof. Let $\omega_{s}(t)=\omega(s t)$. Then $\omega_{s} \cdot \omega_{s}^{-1}$ is a homotopy of a loop to the constant. Let $s_{0}=\sup \left\{s \in I \mid \tau\left(\omega_{t} \cdot \omega_{t}^{-1}\right)=e\right.$ for $\left.t \leqq s\right\}$. By Lemma 1 $s_{0}>0$; we shall show by a contradiction that $s_{0}=1$.

If $s_{0}<1$, then $\omega_{s_{0}} \cdot \omega^{\prime}$ is a reparametrization of $\omega$ where $\omega^{\prime}(t)$ $=\omega\left(s_{0}+\left(1-s_{0}\right) t\right)$. Consider $\omega_{s}^{\prime}(t)=\omega^{\prime}(s t)$ and $\tau\left(\omega_{s}^{\prime} \cdot \omega_{s}^{\prime-1}\right)$. Lemma 1 gives us $s_{1}>0$ such that $\tau\left(\omega_{s}^{\prime} \cdot \omega_{s}^{\prime-1}\right)=e$ for $s<s_{1}$. However $\omega_{s_{0}} \cdot \omega_{r}^{\prime}$ is a reparametrization of $\omega_{s_{0}-r-r s_{0}}$. Thus

$$
\begin{aligned}
\tau\left(\omega_{s_{0}+r-r s_{0}} \cdot \omega_{s_{0}+r-r s_{0}}^{-1}\right) & =\tau\left(\left(\omega_{s_{0}} \cdot \omega_{r}^{\prime}\right) \cdot\left(\omega_{s_{0}} \cdot \omega_{r}^{\prime}\right)^{-1}\right) \\
& =\tau\left(\omega_{s_{0}}^{-1}\right) \circ \tau\left(\omega_{r}^{\prime-1}\right) \circ \tau\left(\omega_{r}^{\prime}\right) \circ \tau\left(\omega_{s_{0}}\right) \\
& =e \text { the identity for } r \leqq s_{1} .
\end{aligned}
$$

Hence $\tau\left(\omega_{s} \cdot \omega_{s}^{-1}\right)=e$ for $s \leqq s_{0}+s_{1}-s_{0} s_{1}$. Since $s_{0}$ is maximal we have 
$s_{0}+s_{1}-s_{0} s_{1} \leqq s_{0}$ or $1 \leqq s_{0}$ which contradicts $s_{0}<1$. q.e.d.

We are now in a position to prove the theorem. The technique is similar to the proof of Cauchy's theorem. Let $\Delta$ denote the model two-simplex and $\Delta_{i}^{n}$ the $i$ th simplex of the $n$th barycentric subdivision. Choose loops $\rho_{n, i}$ which are (clockwise) homeomorphisms of the reals modulo 1 onto the boundary of $\Delta_{1}^{n}$. By the convexity of $\Delta$ define the path $\rho_{x, n, i}(t)=(1-t) x+t \rho_{n, i}(0)$ where $x \in \Delta$. Let $\partial_{x} \Delta_{i}^{n}$ denote the loop $\rho_{x, n, i} \cdot\left(\rho_{n, i} \cdot \rho_{x, n, i}^{-1}\right)$.

ProOF OF ThEOREM BY CONTRAdiction. Let $\omega$ be a null homotopic loop such that $\tau(\omega) \neq e$ the identity. Let $\sigma: \Delta \rightarrow B$ be a singular simplex such that $\sigma \circ \partial_{0} \Delta=\omega$. There must be a simplex $\Delta_{i_{1}}^{\prime}$ of the first barycentric subdivision such that $\tau\left(\sigma \circ \partial_{0} \Delta_{i_{1}}^{\prime}\right) \neq e$ for otherwise $\tau(\omega)=e$. We continue by induction and find a nested sequence of simplexes $\Delta_{i_{n}}^{n}$ such that $\tau\left(\sigma \circ \partial_{0} \Delta_{i_{n}}^{n}\right) \neq e$. Let $x=\bigcap_{n=0}^{\infty} \Delta_{i_{n}}^{n}$. Let $\rho_{s}(t)$ be a homotopy of loops at $x$ such that $\rho_{1 / n}=\partial_{x} \Delta_{i_{n}}^{n}$. Then by Lemma 1 there exists $s_{0}>0$ such that $\tau\left(\sigma \circ \rho_{s}\right)=e$ for $s \leqq s_{0}$; in particular there is some $n$ such that $\tau\left(\sigma \circ \partial_{x} \Delta_{i_{n}}^{n}\right)=e$. But this implies $\tau\left(\sigma \circ \partial_{0} \Delta_{i_{n}}^{n}\right)=e$ which is the desired contradiction. q.e.d.

THEOREM. A bundle over a finite polyhedron with a structural group $G$, which has no small subgroups, has a transitive translation function if and only if it is equivalent in $G$ to an $H$ bundle where $H$ is a totally disconnected subgroup of $G$.

Proof. Let $\tau$ be the translation function that exists by the Hurewicz uniformization theorem for the $H$ bundle. Then since the maps $\tau\left(\omega_{1} \cdot \omega_{2}\right)$ and $\tau\left(\omega_{2}\right) \circ \tau\left(\omega_{1}\right)$ are homotopic and $H$ is totally disconnected it follows that they are equal. Hence $\tau$ is transitive.

If, on the other hand, there is a transitive translation function $\tau$, we can construct a coordinate bundle with a totally disconnected group. Select a point $b_{0}$ in the base space and let $\phi_{0}\left(b_{0}\right): Y \rightarrow p^{-1}\left(b_{0}\right)$ be one of the coordinate maps restricted to $Y \times b_{0}$. For every vertex $a_{i}$ select a contraction of its star to the point $b_{0}$. Thus for every $x \in$ St $a_{i}$ we obtain a path $\omega_{a_{i}, x}$ from $b_{0}$ to $x$. Define the maps $\phi_{a_{i}}: Y \times$ St $a_{i} \rightarrow p^{-1}\left(\right.$ St $\left.a_{i}\right)$ by $\phi_{a_{i}}(y, x)=\tau\left(\phi_{0}(y), \omega_{a_{i}, x}\right)$. Thus we obtain coordinate maps which are compatible with the original coordinate maps. The subgroup $H$ of $G$ which is spanned by the $g_{a_{i}, a_{j}}(x)$, $x \in \mathrm{St} a_{i} \cap \mathrm{St} a_{j}$, is the continuous image of the finitely generated fundamental group of the base space and hence $H$ is totally disconnected. q.e.d. 